5-1-2019

\title{
Mitigating energy loss in a robot hopping on a physically emulated dissipative substrate
}

Sonia F. Roberts

University of Pennsylvania, soro@seas.upenn.edu

Daniel E. Koditschek

Electrical and Systems Engineering, University of Pennsylvania

Follow this and additional works at: https://repository.upenn.edu/ese_papers

Part of the Electrical and Computer Engineering Commons, and the Systems Engineering Commons

\section{Recommended Citation}

Sonia F. Roberts and Daniel E. Koditschek, "Mitigating energy loss in a robot hopping on a physically emulated dissipative substrate", ICRA 2019. May 2019. 


\title{
Mitigating energy loss in a robot hopping on a physically emulated dissipative substrate
}

\begin{abstract}
We work with geoscientists studying erosion and desertification to improve the spatial and temporal resolution of their data collection over long transects in difficult realworld environments such as deserts. The Minitaur robot, which can run quickly over uneven terrain and use a single leg to measure relevant ground properties such as stiffness, is an attractive scout robot candidate for inclusion in a heterogeneous team in collaboration with a heavily geared, sensor-laden RHex. However, Minitaur is challenged by long-distance locomotion on sand dunes. Previous simulation results suggested that the energetic cost of transport can be mitigated by programming a virtual damping force to slow the intrusion of a Minitaur foot into simulated granular media following a bulk-behavior force law. In this paper, we present a ground emulator that can be used to test such locomotion hypotheses with a physical single-legged hopper jumping on emulated ground programmed to exhibit any compliance and damping characteristics of interest. The new emulator allows us to corroborate the conclusions of our previous simulation with physical hopping experiments. Programming the substrate emulator to exhibit the mechanics of a simplified bulk-behavior model of granular media characterized by linear stiffness and quadratic damping, we achieve a consistent energy savings of $20 \%$ in comparison with a nominal controller, with savings of up to $50 \%$ under specific conditions.
\end{abstract}

For more information, see https://kodlab.seas.upenn.edu.

\section{Disciplines}

Electrical and Computer Engineering | Engineering | Systems Engineering 


\title{
Mitigating energy loss in a robot hopping on a physically emulated dissipative substrate
}

\author{
Sonia Roberts ${ }^{1}$ and Daniel E. Koditschek ${ }^{1}$
}

\begin{abstract}
We work with geoscientists studying erosion and desertification to improve the spatial and temporal resolution of their data collection over long transects in difficult realworld environments such as deserts [1]. The Minitaur [2] robot, which can run quickly over uneven terrain and use a single leg to measure relevant ground properties such as stiffness [3], is an attractive scout robot candidate for inclusion in a heterogeneous team in collaboration with a heavily geared, sensor-laden RHex [4]. However, Minitaur is challenged by long-distance locomotion on sand dunes. Previous simulation results [5] suggested that the energetic cost of transport can be mitigated by programming a virtual damping force to slow the intrusion of a Minitaur foot into simulated granular media following a bulk-behavior force law [6]. In this paper, we present a ground emulator that can be used to test such locomotion hypotheses with a physical single-legged hopper jumping on emulated ground programmed to exhibit any compliance and damping characteristics of interest. The new emulator allows us to corroborate the conclusions of our previous simulation with physical hopping experiments. Programming the substrate emulator to exhibit the mechanics of a simplified bulk-behavior model of granular media characterized by linear stiffness and quadratic damping, we achieve a consistent energy savings of $20 \%$ in comparison with a nominal controller, with savings of up to $50 \%$ under specific conditions.
\end{abstract}

\section{INTRODUCTION}

\section{A. Contribution}

This paper introduces a simple, robust physical substrate emulator that can be mechanically coupled to a one degreeof-freedom robot leg for vertical hopping experiments on "ground" with an arbitrary force-extension law for an arbitrary number of jumps. We use this ground emulator to test a locomotion hypothesis about virtual energy dissipation developed in simulation [5], and support the previous conclusion that virtual energy dissipation can be used to mitigate electromechanical energy loss to substrates exhibiting granular media mechanics.

\section{B. Bulk-behavior models of granular media in response to locomotion}

Extensive research on the bulk behavior of granular media in response to intrusion by robot and animal limbs [6], [7] has culminated in an added-mass model which is robust and reliable enough to be used for optimal control [8] and Gaussian-based online learning [9]. Three forces comprise the added-mass model: A stiffness function of depth, $k_{g}(x)$, which describes the hydrostatic-like forces from the granular media in response to intrusion and scales with the surface

\footnotetext{
${ }^{1}$ Electrical and Systems Engineering, University of Pennsylvania, Philadelphia, PA. Contact: soro at seas dot upenn dot edu
}

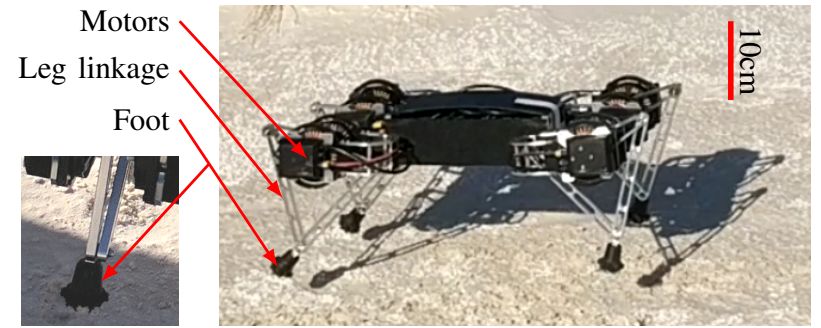

Fig. 1: Minitaur, the target robot under study, in an interdune area at White Sands National Monument in 2017. The compact interdunes were the only areas where Minitaur could run.

area of the intruding face; an inertial drag energy dissipation function of depth and velocity, $d_{g}(x) \dot{x}^{2}$, which describes the hydrodynamic-like forces from the inertia of individual grains recruited to move along with the intruding limb; and a force from the added mass of the grains accelerated along with the intruding limb, $m_{a}(x) \ddot{x}$. All three functions are initially nonlinear during the formation of this "cone" of added grains accelerated with the intruding limb. Once the cone is fully formed, the depth-dependent compliance becomes linear and the damping becomes quadratic. The depth at which the cone is fully formed depends on the surface area of the intruding foot.

These powerful results require the use of large fluidizable beds of idealized and well studied granular media (for example, poppyseeds), which must be prepared in between each experiment and may only be used for either experiments on a single vertical jump [6] or forward locomotion [10].

\section{A Minitaur limb can measure ground erodibility}

Minitaur is a quadrupedal direct-drive robot with legs that can be programmed for locomotion using parallel compositions of virtual damped linear springs [11], [12]. (See Figure 1). A single Minitaur leg can also be used to sense ground properties relevant to erodibility such as shear stress using trajectory control [3]. However, as the robot is challenged by long-distance locomotion on highly dissipative, unidirectionally compliant ground like sand, presently all ground measurement experiments are conducted by a single Minitaur leg carried on the back of a heavily geared RHex [1]. By increasing the robot's ability to carry its own weight under these conditions, we aim to operate Minitaur as a "scout" robot in a heterogeneous robot team along with the "pack mule" RHex carrying other scientific equipment. 


\section{Compositions of simple controllers for locomotion}

Reliable, robust control for hoppers using compositions of simple controllers was proposed originally by Raibert in 1986 [13]. A compression-extension control law is a simple controller for a single jumping leg introduced by Raibert, modeled as a mass (body) on a spring (leg). Under this control law, the programmed leg spring switches between a soft and a stiff compliance gain, causing the robot to switch between two modes of locomotion: stance and flight. During the first half of stance, the robot compresses its soft programmed leg spring. When the leg is fully compressed and the body velocity (or change in leg length) goes to zero, the virtual leg spring stiffness is instantaneously increased, injecting a quantity of potential energy into the leg spring. The potential energy stored in the stiff leg spring accelerates the body mass up. Once the robot's leg returns to its set neutral length, its programmed compliance is reset to its soft gain. As the body mass continues its upward trajectory, the robot enters flight mode, and is ready to compress its soft leg spring again when it touches down at the end of flight.

To create a hopper that runs forward and backward rather than simply jumping up and down, this simple compressionextension controller for vertical hopping can be composed with a fore-aft controller that uses the body pitch angle to control speed [13]. By composing each pair of two legs in the front and back of the robot into two virtual leg springs, and controlling the relative height of the two virtual leg springs to alter body pitch, a quadrupedal machine can be programmed to perform a very simple and robust bounding gait [2] with readily verified stability guarantees [11].

In this contribution, we suggest a modification to the vertical hopping factor of the parallel controller composition that significantly reduces the energetic cost of transport. The reduction in energetic cost comes from attending to the fact that the virtual leg springs in a direct-drive machine [2] are only emulating the storage of mechanical energy via appropriately controlled flows of electrical power.

E. Active damping for stability, control, and energy loss mitigation in virtual leg springs

To mitigate the loss of mechanical energy to the ground, we propose adding a virtual active damping function to the stiff virtual leg spring in proportion to the speed of the foot's intrusion into the ground. For $x_{b}, x_{f}$ the positions of the robot's body and foot respectively (see Figure 2), the active damping function takes the form $F_{A D}=-\left(d_{A D} \dot{x}_{f}\right) \cdot\left(\dot{x}_{b}-\right.$ $\left.\dot{x}_{f}\right)$, and is added to the stiff virtual leg spring force during the second half of the hopper's stance mode.

The use of a damper to stabilize hopping height on rigid ground was first described in [14], and was applied in an empirically demonstrated series of higher degree-of-freedom compositions in [11]. The use of an active damping function to mitigate energy losses to unidirectionally compliant substrates was first described in [5], which used simulations of a one-legged hopper jumping on granular media under the added-mass model [6] to explore the energetic cost of jumping to various heights on ground with a variety of

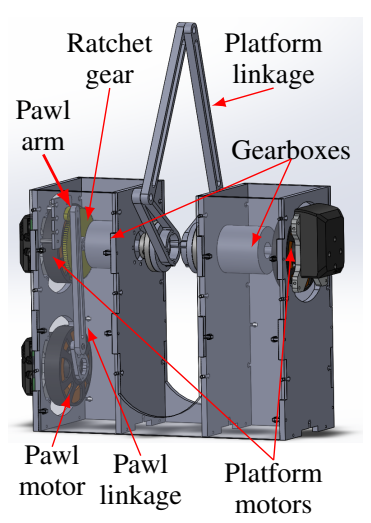

(a)

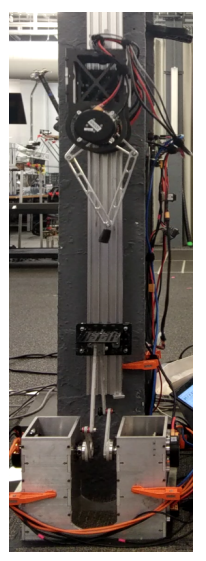

(b)
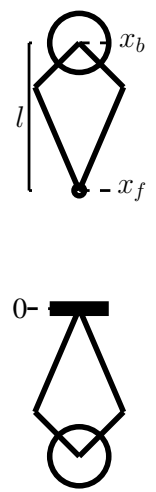

(c)
Fig. 2: (a) A semi-transparent CAD model of the ground emulator shows the ratchet gear (yellow) attached to one of the motors controlling the leg that moves the platform up and down on the linear rail. A separate motor module controlled the ratchet's pawl arm through a linkage. (b) The ground emulator and hopper. (c) A schematic of the hopper and the ground emulator.

stiffnesses and dissipation functions. We suggested using these simulations that dissipating energy "stored" in a virtual leg spring to a virtual damper before the energy is transferred to a unidirectionally coupled physical system where it would be dissipated mechanically with no return could significantly reduce the energetic cost of vertical jumping, as measured in joules drawn from a battery. We found a consistent $20 \%$ savings with the active damping controller when jumping on ground with a variety of different stiffness and damping coefficients and a variety of touchdown velocities [5].

\section{METHODS}

\section{A. Simplified bulk-behavior model of granular media as a unidirectional Hooke's law spring with quadratic damping}

During locomotion on granular media, stance under a compression-extension controller occurs in two stages: First, the soft virtual leg spring compresses under the weight of the body mass, simultaneously pushing the foot into the ground. In previous simulations of single Minitaur legs hopping on granular media, the "cone" of added grains accelerated underneath the robot's foot was quickly formed during this time [5] (see Section I-B).

When the robot's leg length stops decreasing, the virtual leg spring created by the PD controller on the leg motors switches to its stiff gain, causing the leg to extend and the robot to jump. The cone of grains is already fully formed at this point, and the compliance and damping functions of the ground's force profile are already in their linear and quadratic regions, respectively. Since the compared controllers only differ during the extension mode, and the nonlinearities in the stiffness and dissipation functions occur during the compression mode, we did not model the nonlinearities. We modeled the ground's stiffness function $k_{g}(x)$ as a Hooke's law spring with no restoring forces, $k_{g}(x)=k x \quad(\dot{x} \leq 0)$, 

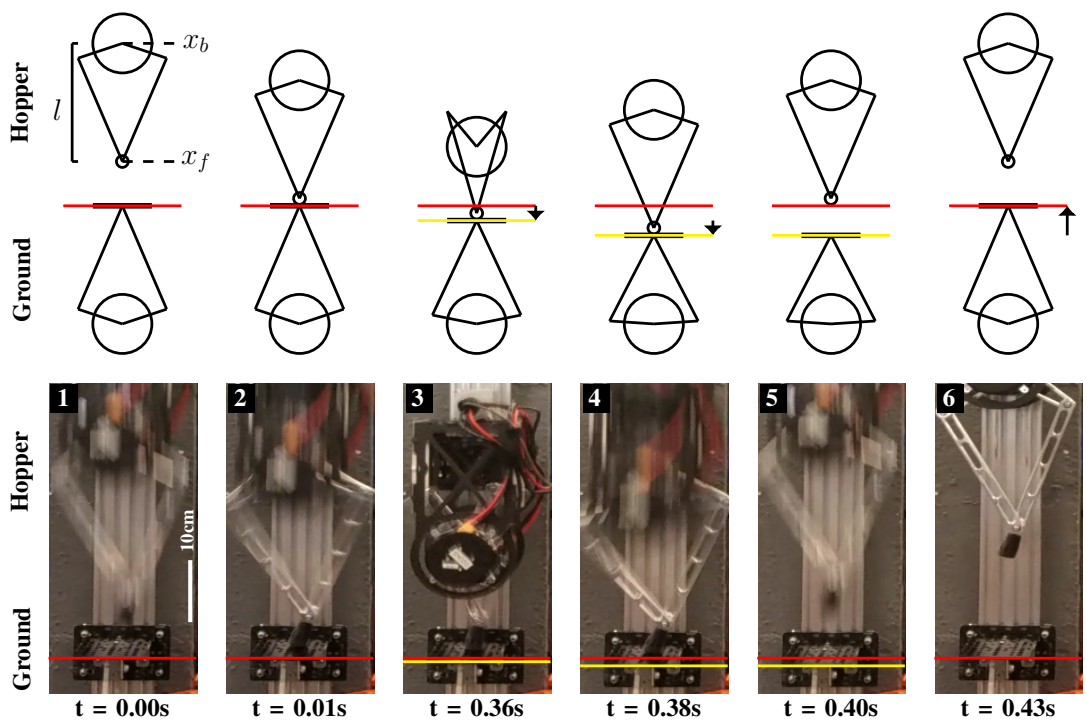

(a) Video stills and schematic demonstrating the behavior of the system.
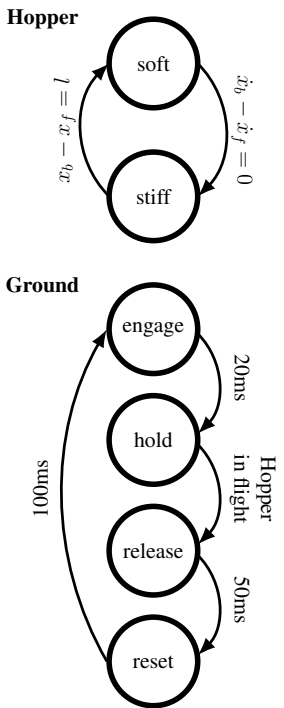

(b) State machines.

Fig. 3: (a) This sequence of video stills and corresponding schematic images shows how the hopper and ground emulator interact during a single jump. The red line shows the neutral point of the ground emulator, and the yellow line shows the displacement of the emulator's platform. (1) The ground emulator engages its ratchet while the hopper is in flight. (2) The hopper touches down. (3) The hopper's soft leg spring compresses easily. Forces exerted by the soft leg spring through the hopper's toe compress the ground emulator and move the platform. (4) The hopper's leg stiffness increases, pushing both the hopper's body up and the platform further down. (5) When the hopper lifts off, the ratchet is still engaged, preventing the ground emulator from exerting restoring forces. (6) While the hopper is in flight, the ground emulator releases its ratchet and resets the platform height to the neutral position. The ground emulator is then ready for the hopper's next jump. (b, top) The hopper's state machine was independent of the ground emulator's, and consisted of only two states: emulating a soft and a stiff virtual leg spring. (b, bottom) The state machine for the ground emulator engaged the ratchet when the hopper was in flight, then lightly held the ratchet's pawl arm down to engage with the gear while the hopper was in stance. When the hopper entered its flight mode, the ground emulator released the ratchet, allowing the platform to reset its position.

and the drag term as a quadratic damping term, $d_{g}(x) \dot{x}^{2}=$ $d \dot{x}^{2}$, with no separately modeled added mass.

\section{B. Physical setup of ground emulator}

The ground emulator used a modified Ghost ${ }^{1}$ Minitaur leg to move a platform up and down a linear rail. ${ }^{2}$ The leg used two opposing T-motor U-8 motors driven by Ghost EtherCAT motor controllers, lightly geared ${ }^{3}$ (4.3:1 reduction) to increase maximum torque while maintaining backdrivability [15]. The body of the emulator and linear rail were clamped to the same vertical I-beam, and both the body of the ground emulator and the vertical rail were leveled in two axes to within 1 degree of inclination. A releasable mechanical stop at the bottom of the vertical rail constrained the range of motion of the leg controlling the platform to prevent the leg from reaching a singularity. The programmed range of motion used for experiments was further restricted to between 20 and $27 \mathrm{~cm}$ (possible range $10-30 \mathrm{~cm}$ ). This is the part of the robot's workspace where it has the least mechanical advantage, and where the mechanical advantage is relatively constant [16]. Because of the consistently low mechanical advantage within this range, the behavior of the limb overall better emulates a linear spring (see Section II-F).

\footnotetext{
${ }^{1}$ Ghost Robotics, 3401 Grays Ferry Ave, Philadelphia, PA 19146 http://www.ghostrobotics.io

${ }^{2}$ OpenBuilds Part Store, https://openbuildspartstore.com

${ }^{3}$ Maxon ceramic planetary gearbox, PN 223081
}

The force-extension behavior produced by the ground emulator is composed of two simple physical actuation subsystems. The lightly geared leg emulates a spring with a linear stiffness function, $F_{s}\left(x_{f}\right)=k x_{f}$, and quadratic damping, $F_{d}\left(\dot{x}_{f}\right)=\alpha \dot{x}_{f}+\beta \dot{x}_{f}^{2}$, with $\alpha$ the native mechanical viscous damping in the system and $\beta$ the programmed quadratic term. The linear damping modeled in coefficient $\alpha$ is due to friction from the linear rail, the 4.3:1 gearboxes, and the ratchet, as well as inefficiencies in the PWM motor controllers. A ratchet gear ${ }^{4}$ attached to one of the two motors controlling the lightly geared leg engages with a pawl arm controlled by a third Ghost motor module (see Figure 2).

\section{Control of ground emulator}

The state machines for the emulator and hopper are both simple, and the hopper's state machine is independent of the ground emulator's (see Figure 3). The ground emulation control entails four states. While the hopper is in flight, the motor controlling the ratchet moves the pawl arm down to engage the gear. Once in position, the motor receives a small constant open-loop command, which produces a small force to hold the pawl arm in place and keep it engaged as the gear rotates. While the emulator is in this state, the hopper enters stance mode, touching down and compressing its leg spring. When its leg spring is fully compressed, the hopper

\footnotetext{
${ }^{4}$ Stock Drive Products/Sterling Instruments, http://sdp-si.com.
} 
switches to a stiff extension spring, and rapidly pushes the emulator's platform down a second time before lifting off and entering its flight mode. The emulator is triggered to release the ratchet when the hopper's leg extension length is close to its rest length, the hopper leg is in its stiff state, and the "hold" state has lasted at least $50 \mathrm{~ms}$, with these conditions serving as a proxy for the hopper being in flight. The release is accomplished by changing the desired position of the platform to slightly below its current position, releasing pressure on the pawl arm, and moving the pawl arm up. Finally, while holding the pawl arm up, the emulator quickly pushes its platform back up to reset the emulated "ground" to its nominal height, and the hopping cycle is ready to repeat. The coefficients for the ground's stiffness and dissipation functions were kept constant over the course of the experiments. The position gain was set to 125 , which corresponds to a stiffness of $387 \mathrm{~N} / \mathrm{m}$, and the velocity gain was set to 10 , corresponding to $53 \mathrm{~kg} / \mathrm{s}$.

\section{Physical setup of hopper}

The one-legged hopper models one quarter of a Ghost Robotics Minitaur, using two opposing motors to control the motion of a toe through a symmetric four-bar linkage. The virtual leg spring force is created using a PD controller on the leg length, and results only from electromechanical forces produced by the motors. The hopper is constrained to move its body center of mass vertically along the same linear rail that constrains the ground emulator's platform. A spring potentiometer attached to the top of the hopper's body measures its height, with values recorded by the microcontroller shared by the hopper and ground emulator. All controlling software was written using the Ghost SDK. Although the emulator and the hopper share a microcontroller, the hopper is powered from its own battery.

\section{E. Control of hopper}

The hopper cycles between two states: emulating a virtual soft leg spring which compresses easily, and emulating a stiff leg spring. When appropriately triggered and reset, the alternation of these two internal hybrid controller modes produce two physical robot modes: Stance, during which time the robot's foot is in contact with the ground; and flight, when it is not. The hopper switches to its stiff programmed leg spring when the leg is compressed beyond a threshold and the velocity of the change in leg length is zero, $\dot{x}_{b}-\dot{x}_{f}=0$. It switches from stiff to soft when the leg again achieves its neutral leg length $l$. The switch from soft spring to stiff injects a large quantity of potential energy when the leg is compressed, causing the robot to jump. See Figure 3.

\section{F. Characterization of ground emulator}

To test whether the ground emulator would exert the programmed forces in the expected ways, we characterized its force response when commanded to emulate a linear spring with linear damping. We generated trajectories through the platform's state space by placing a mass on the platform,

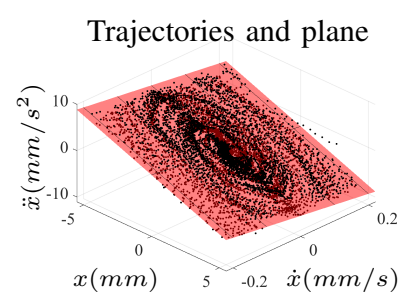

(a)

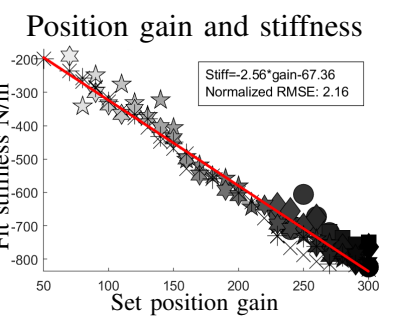

(c)

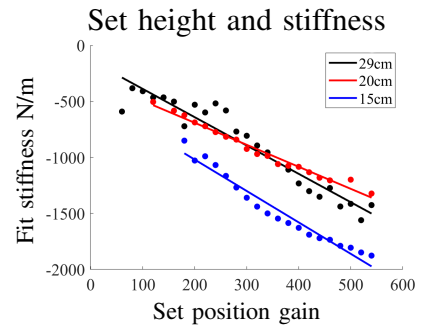

(b)

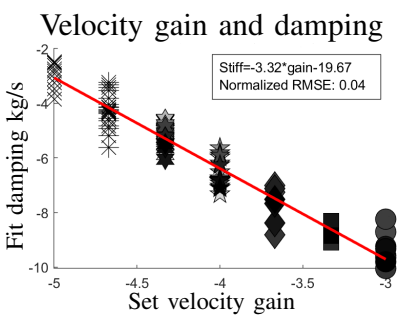

(d)
Fig. 4: (a) Example data of trajectories through the platform's state space that were used to relate the acceleration of the platform to its position and velocity, and thus fit the physical values of the virtual spring constant and damping coefficient. The fitted plane is in red. Multiple mass conditions were used to fit each plane. (b) The fits to physical stiffness were similar when the ground emulator's neutral point was 20 (red) and 29 (black) cm, but the mechanical advantage conferred by the crouched posture when the emulator's linkage was more compressed at $15 \mathrm{~cm}$ (blue) resulted in stiffer physical springs from the same set gains (see [16] for details). (c, d) At neutral length $20 \mathrm{~cm}$, the set position and velocity gains predict the physically measured stiffness and damping well under a linear model. In both plots, different marker shapes correspond to experiments conducted with different velocity gains, and the marker opacity corresponds to experiments conducted with different position gains. Each marker corresponds to one fitted plane (example in (a)).

pushing the platform down to displace the mass, and recording the extension position and velocity of the limb as the platform oscillated and came to rest (see Figure 4.) For these experiments, the ratchet was disengaged, allowing the platform to oscillate up and down. In lieu of a variety of initial conditions, we used a variety of masses to achieve a range of trajectories through the platform's state space. We collected at least ten trajectories under each combination of position gain, velocity gain, and mass.

We calculated the acceleration of the platform at each time step from the extension velocities and time in milliseconds measured through the Ghost SDK at $300 \mathrm{~Hz}$. The force exerted by the virtual leg spring at each time step was then calculated by multiplying the acceleration by the total mass that the leg was pushing. Finally, we used linear regression (pseudoinverse on the position and velocity data) to fit a plane predicting the force. The fitted coefficients on the position and velocity axes were taken as the physical stiffness and damping coefficients of the virtual spring. Planes were only fit to experimental conditions where we had at least 250 data points (Figure 4a).

We characterized the physical stiffness and damping of the emulator with three different set platform heights to ensure 
that we could expect the same physical stiffness in different parts of the workspace. We found little difference between the physical stiffnesses when the emulator's limb was extended to 20 and $29 \mathrm{~cm}$, but the physical stiffness was much greater when the limb was extended to only $15 \mathrm{~cm}$, resulting in a "crouched" posture that confers mechanical advantage [16] (Figure 4b). The physical stiffness and damping of the emulator was found for a range of position and velocity gains, and we used linear regression to predict the physical stiffness and damping from the set gains (Figures $4 c-d$ ). The characterization experiments were performed with an older version of the Ghost motor controllers and firmware, but using the same motors and control software.

\section{G. Compression-extension and active damping controllers}

As our nominal controller, we employed a Raibert-style compression-extension control law [13] (see Section I-D). A soft spring during the first half of stance allows the robot's leg to compress under the weight of its body. When the rate of change of the leg length goes to zero, the controller switches to a stiff spring. This sudden injection of potential energy causes the robot to jump.

In comparison, we tested two versions of the active damping controller proposed in [5]. This control law is very similar to the simple Raibert compression-extension controller, but a damping force is added to the stiff spring in proportion to the velocity of intrusion of the robot's toe into the ground. Here, "intrusion" is considered to be the vertical displacement of the ground emulator's platform. In the constant active damping controller, the same proportional damping gain is used for all stiff spring conditions tested. In the scaled active damping controller, the gain used in the active damping controller was scaled proportionately with the position gain used for the stiff spring.

\section{H. Experiments comparing controllers}

We jumped the hopper on the ground emulator under five different stiff spring position gain conditions (100, 200, $300,400,500)$ using two versions of the active damping controller: one in which the active damping gains were set to the same value (50) for all stiff spring conditions, and one in which the active damping gains scaled linearly with the stiff spring's position gain at a ratio of 1:6. For example, for position gain 200, the scaled active damping gain was $\frac{200}{6}=33$. These values coincided for the condition with stiffness gain $300(=6 \cdot 50)$.

The experiments reported here were run over four nonconsecutive days. On days where many experiments were run, the trial ordering was randomized. On days with only a few experiments, block ordering was used to prevent any potential spurious correlations between time and position gains or controller type (for example, due to heating of the motor windings or changing friction in the gears or linear rail). Every day that experiments were run, at least one control experiment for each extension condition was run using the nominal compression-extension controller.

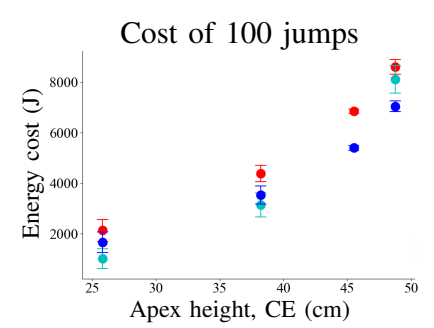

(a)

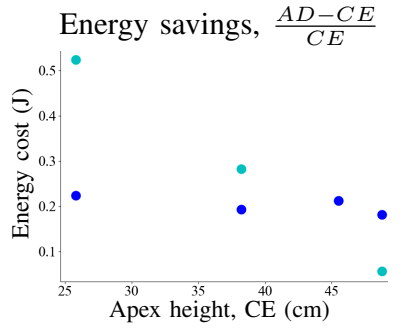

(b)
Fig. 5: (a) The compression-extension controller (CE, red) consistently required more energy from its battery than the constant (cyan) or scaled (blue) active damping controllers (AD) to jump 100 times. All error bars are the standard error of the mean. (b) The difference between the mean cost of 100 jumps for the constant (cyan) and scaled (blue) AD conrollers is plotted for different apex heights. Under a constant active damping controller, the percent energy savings scales linearly with jump height, but when the active damping gain scales, the percent savings is a consistent $20 \%$.

The protocol for each experiment was as follows. First, a fully charged 4-cell lithium ion polymer battery ${ }^{5}$ was connected to the hopper. The ground emulator and microcontroller were connected to a separate power supply and turned on. The ground emulator moved the platform to its neutral position and the hopper was lowered by hand until its toe just touched the platform. The hopper was then released, allowing its body mass to compress the soft leg spring and trigger a switch to the stiff leg spring, causing the robot to take its first jump. The hopper was allowed to complete 100 jumps before being caught and turned off. Between four and seven repetitions of each experiment were conducted.

The battery was then recharged using a ThunderPower charger, and the mAh reported by the charger was recorded. We assumed a full battery voltage of 16.8 and calculated the joules drawn from the battery as the energy cost for the 100 jumps. In addition to the data recorded from the charger, some control and validation data (gains, current states, and positions and velocities of the hopper and ground emulator) was logged at $300 \mathrm{~Hz}$ through a serial port connection to the microcontroller and at least one 60fps video was taken of each experimental condition. The only data logged through the serial port reported here is the apex heights for the different conditions, which were recorded using a spring potentiometer attached to the hopper.

\section{ANALYSIS AND RESULTS}

\section{A. Active damping controller reduces energy cost for a variety of jump heights}

The constant and scaled active damping controllers both consistently used less energy than the compression-extension controller over 100 jumps, but exhibited different patterns of performance relative to the compression-extension controller (see Figure 5). For each of the two active damping controllers, we calculated the percent improvement provided by

\footnotetext{
${ }^{5}$ ThunderPower, Reaper series, $7200 \mathrm{mAh}$
} 


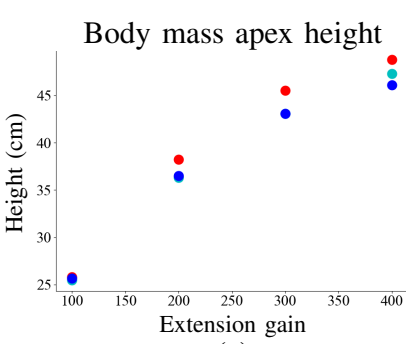

(a) (b)

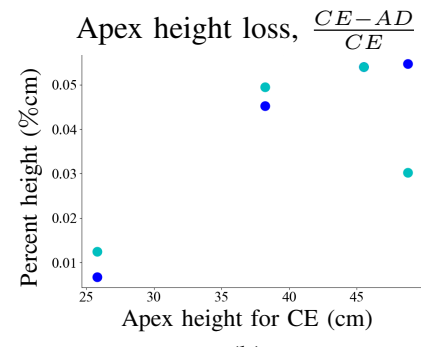

Fig. 6: (a) The body mass apex height increased approximately linearly as the extension gain was increased. (b) The percent loss in apex height, calculated using the average apex heights, between the two active damping (AD) and simple compression-extension (CE) conditions was consistently less than 6\%. In both plots, red indicates the compression-extension controller, cyan the active damping controller with the same active damping gain for different extension stiffnesses, and blue the active damping controller with an active damping gain that scales with the extension stiffness. These values coincide for extension gain 300 (apex height $45 \mathrm{~cm}$ ). Each sample consists of between 200 and 700 apex heights.

the active damping (AD) controller over the compressionextension (CE) controller, that is, the percent of the joules cost for 100 jumps with the compression-extension controller that was saved by using the active damping controller: $\frac{C E-A D}{C E}$. The constant active damping controller, which added the same damping force relative to the robot's intrusion velocity regardless of the stiffness of the extension leg spring, gave extremely high relative improvement in the lowest stiffness condition and very little improvement in the highest stiffness condition, with an approximately linear relationship to apex jump height. The scaled active damping controller, in which the active damping gain scaled linearly with the hopper's extension spring gain, gave a consistent $20 \%$ improvement across all jump height conditions. For low extension mode stiffness gains, the constant active damping controller dissipates more energy into the virtual leg spring than the scaled active damping controller, suggesting that even more energy might be saved across different conditions by using a larger scaled active damping gain.

\section{B. Energy savings from active damping controller come with little cost to apex jump height}

The apex center of body mass heights were determined by the spring potentiometer readings logged over a serial communications port to the microcontroller. Noting that there was no appreciable difference in jump height between the two stiffest conditions (gains $=400$ and 500), we determined that the hopper's motors were already exerting their maximum torque in the second-stiffest condition and excluded the stiffest condition from further analysis.

Apex heights were determined to be the local maxima of the lightly smoothed spring potentiometer data (moving average over 10 samples), excluding the first two hops of each experiment and any hops where a dropped data packet obscured part of the aerial phase of the hop. Because all experimental conditions had at least 200 data points, and jump heights were very regular, standard deviations were very small $(<0.75 \mathrm{~cm}$ for all conditions) and are not plotted. The apex heights varied approximately linearly with the extension position gain for all experimental conditions (Figure 6), and the percent difference between the apex center of body mass for both active damping conditions and the control condition was consistently less than $6 \%$.

\section{CONCLUSIONS}

We have introduced a ground emulator that can be used to test hypotheses about locomotion on dissipative compliant ground with arbitrary force extension laws, including generalized granular media models. We used this system to perform some preliminary tests in emulation of a hypothesis developed originally in simulation [5], that is, that the dissipation of virtual energy into a virtual damper in an electromechanical system before it is transferred to a nonlinear, unidirectional, and highly dissipative mechanical system can significantly reduce the energetic cost of transport.

As the neutral leg length for the hopper was set to $25 \mathrm{~cm}$, which is very close to the lowest apex jump height of $25.6 \mathrm{~cm}$, the lowest apex height condition represented a very modest "jump" which would be appropriate to use in the stance portion of a step in a walking gait. The highest apex height, $49.3 \mathrm{~cm}$, represented a large jump that might be used to climb up a steep slope or run quickly over a long distance when composed with other controllers for steering and gait selection (see Section I-D). The consistent 20\% energy savings and low $(<6 \%)$ cost to apex height across this large range of jump heights suggest that dissipating energy using virtual forces in electromechanical systems has potential to mitigate energy costs in a large variety of locomotion applications on dissipative ground.

Analysis now under way will attempt to provide a formal explanation for these empirically revealed patterns of savings, and, in particular, consider whether and why the scaled active damper achieves superior savings at lower energy levels. We anticipate that the incorporation of active damping into the vertical hopping controller for a direct-drive robot programmed using compositions of damped springs could significantly reduce the energetic cost of transport in environments where locomotion on highly dissipative ground is necessary, including our own application of research in deserts and other natural soils. While the effect of a gearbox on the energy savings is still undetermined, any robot that uses impedance control to interact with dissipative substrates could potentially gain a large energy savings by implementing a virtual damper that dissipates energy in proportion to the intrusion velocity of its end effector.

\section{ACKNOWLEDGMENT}

This work was supported by NSF INSPIRE grant 1514882 and in part by NSF NRI-2.0 grant 1734355 . We thank Ghost Robotics, and in particular Avik De, Tom Jacobs, Gavin Kenneally, and Turner Topping for technical support; Diedra Krieger for administrative support; and Terry Kientz and Jeremy Wang for machining support. 


\section{REFERENCES}

[1] F. Qian, D. Jerolmack, N. Lancaster, G. Nikolich, P. Reverdy, S. F. Roberts, T. Shipley, R. S. Van Pelt, T. M. Zobeck, and D. E. Koditschek, "Ground robotic measurement of aeolian processes," Aeolian Research, vol. 27, pp. 1-11, 2017.

[2] G. Kenneally, A. De, and D. E. Koditschek, "Design principles for a family of direct-drive legged robots," IEEE Robotics and Automation Letters, vol. 1, no. 2, pp. 900-907, 2016.

[3] F. Qian, D. Lee, G. Nikolich, D. E. Koditschek, and D. Jerolmack, "Rapid in-situ characterization of soil erodibility with a field deployable robot," Journal of Geophysical Research (submitted), 2019.

[4] G. C. Haynes, J. Pusey, R. Knopf, A. M. Johnson, and D. E. Koditschek, "Laboratory on legs: an architecture for adjustable morphology with legged robots," in Unmanned Systems Technology XIV, vol. 8387, p. 83870W, International Society for Optics and Photonics, 2012.

[5] S. Roberts and D. E. Koditschek, "Reactive velocity control reduces energetic cost of jumping with a virtual leg spring on simulated granular media," in 2018 IEEE International Conference on Robotics and Biomimetics (submitted), IEEE, 2018.

[6] J. J. Aguilar and D. I. Goldman, "Robophysical study of jumping dynamics on granular media," Nature Physics, vol. 12, no. 3, p. 278, 2016.

[7] C. Li, T. Zhang, and D. I. Goldman, "A terradynamics of legged locomotion on granular media," Science, vol. 339, no. 6126, pp. 1408$1412,2013$.

[8] C. M. Hubicki, J. J. Aguilar, D. I. Goldman, and A. D. Ames, "Tractable terrain-aware motion planning on granular media: an impulsive jumping study," in Intelligent Robots and Systems (IROS), 2016 IEEE/RSJ International Conference on, pp. 3887-3892, IEEE, 2016.

[9] A. H. Chang, C. M. Hubicki, J. J. Aguilar, D. I. Goldman, A. D. Ames, and P. A. Vela, "Learning to jump in granular media: Unifying optimal control synthesis with Gaussian process-based regression," in Robotics and Automation (ICRA), 2017 IEEE International Conference on, pp. 2154-2160, IEEE, 2017.

[10] F. Qian, T. Zhang, W. Korff, P. B. Umbanhowar, R. J. Full, and D. I. Goldman, "Principles of appendage design in robots and animals determining terradynamic performance on flowable ground," Bioinspiration \& biomimetics, vol. 10, no. 5, p. 056014, 2015.

[11] A. De and D. E. Koditschek, "Vertical hopper compositions for preflexive and feedback-stabilized quadrupedal bounding, pacing, pronking, and trotting," The International Journal of Robotics Research, vol. 37, no. 7 , p. 743778,2018

[12] A. De and D. E. Koditschek, "Parallel composition of templates for tail-energized planar hopping," in Robotics and Automation (ICRA), 2015 IEEE International Conference on, pp. 4562-4569, IEEE, 2015.

[13] M. H. Raibert, Legged robots that balance. MIT press, 1986.

[14] G. Seçer and U. Saranl,, "Control of monopedal running through tunable damping," in Signal Processing and Communications Applications Conference (SIU), 2013 21st, pp. 1-4, IEEE, 2013.

[15] G. Kenneally, W.-H. Chen, and D. E. Koditschek, "Actuator transparency and the energetic cost of proprioception," ISER, 2018 (to appear).

[16] G. Kenneally and D. E. Koditschek, "Leg design for energy management in an electromechanical robot," in 2015 IEEE/RSJ International Conference on Intelligent Robots and Systems (IROS), pp. 5712-5718, IEEE, 2015. 